\title{
Findings from an Assessment of State Title V Workforce Development Needs
}

\begin{abstract}
Objective To describe results of a 2008 assessment of Title V workforce competencies and training needs at the state level, and examine preferences and barriers related to available education and training opportunities.

Methods A web-based survey was administered May through August, 2008 to Maternal and Child Health (MCH) and Children and Youth with Special Health Care Needs (CYSHCN) program leaders in all 50 states, and U.S. jurisdictions. Forty-nine MCH (96\%) and 44 CYSHCN (86\%) programs and four territories completed surveys. A major focus of the survey related to competencies in six core domains: Public Health/Title V Knowledge Base, Communication, Critical Thinking, Management Skills, Family Centered Care and Medical Home, and Leadership Development.

Results The top training needs identified by state Title V programs fall into the global category of critical thinking, including skills in $\mathrm{MCH}$ data synthesis and translation, in program evaluation, and in systems thinking. The need to enhance personal rather than organizational leadership skills was emphasized. Blended learning approaches (graduate education), and national conferences with skills building workshops (continuing education) were identified as preferred training modalities. Barriers to training included lack of career opportunities, insufficient agency support, and inability to take leave (graduate education), and travel restrictions, release time limitations, costs, and limited geographic access (continuing education). Both the focus of training and preferred training modalities differed from previous $\mathrm{MCH}$ workforce survey findings.

Conclusion Given the changing needs expressed by state Title V leaders as well as their training preferences, it is important that current and future graduate education and continuing education approaches be better aligned to meet these needs and preferences.
\end{abstract}

Keywords: Title V programs, workforce, continuing education, training, graduate education 


\section{Findings from an Assessment of State Title V Workforce Development Needs}

\section{BACKGROUND}

State Title V programs charged under the 1935 Social Security Act with responsibility for ensuring the health of all mothers and children have a respected track record of addressing the needs of the Maternal and Child Health $(\mathrm{MCH})$ population, identifying and responding to emerging trends with a focus on prevention, and developing systems of care. In fact, $\mathrm{MCH}$ is a substantial portion of the work of public health agencies, particularly at the local and state levels. The activities and programs that constitute the Title V program vary across states and regions. In any given jurisdiction, the scope of program activities is configured to best address the population needs and resources in that state.

A focus on $\mathrm{MCH}$ workforce needs is made urgent in a severe economic downturn, with state budgets strained and public health agencies furloughing staff that are already stretched to their limits. State public health agencies and their Title V programs are being substantially downsized as a result of unparalleled state deficits at the very same time that they are facing the dilemma of an aging workforce. (1-4) Decreasing the size of government translates into movement of personnel across units of executive branch agencies to fill program vacancies, often contributing to the deprofessionalization of these health agencies. In fact, a recent study indicates that 80 percent of public health workers have not received formal training in their specific job functions. (5)

Together, these phenomena indicate an urgent need to equip the people currently working in state MCH programs and those who will be entering government service in public health or related agencies with the knowledge and skills necessary to continue in their effective stewardship of the MCH population. 
9-23-10

\section{Specific Workforce Development Context for MCH/Children and Youth with Special Health Care Needs (CYSHCN)}

The Maternal and Child Health Block Grants to states and the MCH Training Program are complementary aspects of a single, enduring focus on the nation's women, children, youth and families (Social Security Act, Title V). In order to improve the health status of women, infants, children, youth and their families, the Health Resources and Services Administration’s Maternal and Child Health Bureau (MCHB) has been investing in the education of MCH professionals since its inception. The Children's Bureau, the precursor to today’s MCHB, supported the continuing education of public health nurses. Starting in the 1940s, the Children’s Bureau supported long term training grants to Schools of Public Health to promote an MCH concentration in their curricula. Over the next several decades, the MCHB Training Program supported clinical training programs as well, nurturing the development of fields such as maternal and pediatric nursing, pediatric cardiology, MCH public health, MCH social work, public health nutrition, adolescent medicine, pediatric dentistry, developmental disabilities, pediatric pulmonology, and developmental-behavioral pediatrics.

The current foci of the MCH training program are: 1) graduate-level MCH professions training; 2) developing the existing workforce; and 3) sustaining a community of MCH professionals. MCHB Training grants support trainees who show promise to become leaders in the MCH field in the areas of teaching, research, clinical practice, and/or administration and policymaking, and faculty who mentor trainees and students in exemplary $\mathrm{MCH}$ public health practice, advance the field through research and dissemination of findings, and develop curricula particular to $\mathrm{MCH}$ and public health. In addition to supporting graduate training of MCH professionals, MCHB funds continuing education programs for practicing $\mathrm{MCH}$ professionals using a variety of delivery methods so that they can address new and emerging issues in the field.

\section{Prior Assessments of Title V Workforce Needs}


Over the years, there have been a number of efforts to document and address MCH workforce needs. A 1992 survey of state MCH and CYSHCN programs conducted by the Association of $\mathrm{MCH}$ Programs (AMCHP) found that state MCH programs’ most critical unmet education needs were graduate education in public health, management, and nursing, and non-degree training in management, program development/management, and clinical skills. The greatest barriers to continuing education were restricted travel, lack of staff to cover duties, and budget restrictions. (6) In 2000, another assessment of Title V programs found that graduate training was needed in $\mathrm{MCH}$ epidemiology, public policy, medicine, management/business administration, genetic counseling, health care administration, and dentistry. Major areas of need for continuing education varied among the several groups queried. The top three identified by state Title V Program Managers, for example, were data analysis and interpretation, program evaluation/planning/development, and needs assessment (MCH), and program implementation, management, and performance measurement (CYSHCN). The greatest barriers to graduate and continuing education remained the same as those found in the AMCHP survey a decade earlier. (7)

\section{Aims of the Paper}

In this paper, we report on results of a 2008 assessment of Title V workforce competencies and training needs at the state level. We examine reported needs, preferences and barriers in the context of education and training opportunities currently funded through the MCH Training Program, as well as related MCHB efforts (e.g., resource centers, data institutes). In addition, we discuss implications for needed adjustments to current MCHB public health graduate and continuing education, and opportunities to improve “alignment” between workforce needs and resources.

\section{METHODS}


The 2008 MCH workforce assessment was conducted through an interorganizational partnership in order to identify the current capacity of the $\mathrm{MCH}$ workforce and the need for training to enable states to carry out the 10 essential public health functions as translated for the field of MCH. (8) The AMCHP Workforce Development Committee provided the organizing structure to convene state Title V leaders, MCH faculty in schools of public health, MCHB staff, and staff representatives of the Association of Teachers of Maternal and Child Health (ATMCH), CityMatCH, and ASTHO (the Association of State and Territorial Health Officials) for conceptualizing and guiding the assessment. Technical aspects of survey design and fielding were led by faculty from the Johns Hopkins Bloomberg School of Public Health.

The workforce assessment was designed to elicit standardized information about the specific programmatic and functional foci of the state Title V MCH and CYSHCN programs, leadership tenure and staffing vacancies, and staff needs with respect to competencies in core knowledge and skill areas. Several aspects of graduate and continuing education needs of the state Title V MCH/CYSHCN workforce were explored, including the state agency's staff development capabilities, strategies and challenges, as well as preferred modalities related to staff training and graduate education. $\mathrm{MCH}$ and CYSHCN programs were queried separately.

Assessment items were based on concepts embodied in five competency models developed by ATMCH, MCHB, the Association of Schools of Public Health (ASPH), the Council on Linkages between Academia and Public Health Practice, and by AMCHP’s Capacity Assessment for State Title V Programs (CAST-5). Several assessment items were drawn from the 1992 and 2000 studies in order to document temporal trends. 
A paper version of the assessment was piloted with four states in January/February of 2008. Data generated in the pilot as well as structured feedback from the pilot states informed revisions to the original content, organization, and fielding strategy. The assessment ultimately was fielded as a webbased SurveyMonkey document (Professional Plan - Copyright (C1999-2010 SurveyMonkey. All Rights Reserved.). The assessment -- also made available in print format -- was distributed in May 2008 under cover correspondence signed by AMCHP and was accompanied by Disclosure Statement and a guidance “Tip Sheet.” Those contacted for the assessment were asked to prepare responses using a process that involved staff perspectives across programmatic areas and at line staff and management levels. Efforts to garner a robust response rate were facilitated by AMCHP notices in member correspondence, and personal contacts made by both the Title V Directors chairing the Workforce Development Committee and AMCHP staff to those not responding within a month. Survey administration, and data cleaning, coding and management were housed at the JHU Women's and Children's Health Policy Center. JHU prepared and presented preliminary survey tables to members of the AMCHP Workforce Committee, who advised on interpretation of the data. This project was reviewed and approved by the University of Illinois at Chicago School of Public Health Institutional Review Board.

\section{RESULTS}

One hundred and twelve Title V program leaders received the request to assist with the survey and all 50 states and the District of Columbia provided at least one program response. Of these, 49 MCH (96 percent) and 44 CYSHCN (86 percent) programs responded. Four of the eight U.S. territories also responded, although those responses are not included in this analysis.

Not only do State Title V program activities reflect a broad range of programmatic foci, but staff also undertake an array of public health functional responsibilities. This 2008 workforce survey 
9-23-10

sought to determine the primary areas of staff function, drawing from the list of " 10 essential public health services” and adapted to include a “program management” function.

\section{TABLE 1 ABOUT HERE}

Not surprisingly, program management was reported most frequently by both MCH and CYSHCN staff as a primary area of function. As exhibited in Table 1, the extent of staff engagement in other functional areas reveals basic similarities between both sets of respondents, but some variation between the two programs are observed. For both programs three of the "top 5" domains of function relate to data collection and analysis, and assessment, planning and policy.

\section{Extent of Need for Training of State Level Program Staff}

A core component of the 2008 workforce assessment was to document the most pressing needs to enhance staff skills or knowledge in areas of critical importance to $\mathrm{MCH}$. Competency sets were culled to create a list of 33 skill or knowledge items. Items were conceptually grouped into six domains: Public Health/Title V Knowledge Base, Communication, Critical Thinking, Management Skills, Family Centered Care and Medical Home, and Leadership Development. Within each of these domains, respondents identified up to three areas with the greatest training needs (Table 2). There was substantial consistency between MCH and CYSHCN programs, with the greatest difference found in the area of family-centered care and medical home.

\section{Areas Where Training Needs are Reported to be the Greatest}

State programs also were asked to identify the three domains (of 6) with the greatest need for training (the response option “no need” was provided). For both programs “critical thinking” skills was reported to be the greatest training need, followed by "leadership development.” Some small differences were observed for $3^{\text {rd }}$ and $4^{\text {th }}$ reported frequency in relation to management $(47 \% \mathrm{MCH}$; 45\% CYSHCN) and public health/Title V Knowledge Base (41\% MCH; 45\% CYSHCN).

\section{What PH Agencies/MCH Programs Do to Address Graduate Education and Training Needs}


The majority of MCH and CYSHCN programs (74\%) assess the training needs of their staff at least annually. Training needs assessments are conducted to aid in enhancing the skills and performance of staff members, for general program development and improvement, to evaluate employee performance, and to meet program training requirements. Although Title V programs collect information on staff training needs, their capacity to address these needs is limited: only $1 \%$ of programs reported that they have the capacity to provide appropriate and accessible training in all areas. Most states, however, offer training in some areas (73\%).

Title V programs use several strategies to facilitate training for their state level staff, including providing paid release time (88\%) and covering the costs of registration fees (84\%) and in-state travel (84\%). Another common strategy is providing staff training on site (80\%). Title V programs have fewer strategies in place to facilitate formal graduate education for staff. Many states offer flexible hours to pursue education (71\%) and some provide tuition reimbursement (54\%), but $16 \%$ of states have no strategies in place to support graduate education for staff members.

\section{Barriers State Title V Program Face, and Preferred Graduate Education (GE) and Continuing Education (CE) Modalities}

Graduate education. If given the opportunity to pursue graduate education, both Title V MCH and CYSHCN program staff in most states prefer a blended learning format that incorporates both distance and onsite learning (72\%). Part-time programs are preferred over full-time. However, $\mathrm{MCH}$ and CYSHCN programs report that staff face barriers to graduate education, particularly a lack of career promotion opportunities (55\%), no financial or logistical support from the agency (46\%), and an inability to take time away from work (27\%). Only $11 \%$ of Title V programs report that staff do not experience barriers to graduate education.

\section{TABLE 3 ABOUT HERE}

Training. State Title V programs use continuing education to enhance the knowledge and skills of program staff. The preferred strategies include national conferences with skills workshops (63\%), 
9-23-10

regional meetings (59\%), and 1-3 day intensive trainings (44\%) Title V programs report that the barriers that most often interfere with continuing education for staff are travel restrictions (69\%), difficulty taking time away from work (63\%), and the cost of trainings (61\%). In addition, nearly one quarter of Title V programs report that geographic access is a barrier to continuing education for staff (23\%).

\section{DISCUSSION}

The top training needs identified by state MCH and CYSHCN directors in the 2008 AMCHP workforce survey fall into a global category reflecting a variety of skills related to critical thinking that cut across competency domains. Such skills included, skills in translating data into viable information, skills to enable synthesis and translation of $\mathrm{MCH}$ data for a variety of audiences, skills for designing and conducting program evaluations, as well as systems thinking skills. In addition, there is a strong emphasis on the need to enhance personal as opposed to organizational leadership skills. While these identified needs overlap somewhat with the graduate and continuing education needs identified in the 2000 needs assessment by Alexander et al. (2002), (7) it is notable that the needs identified in the current survey focus less on capacity building for data generation and more on use of data/evidence, synthesis of information, and translation of data into information that shapes policies and programs.

This survey also identified blended learning approaches for graduate education, and national conferences with skill building workshops for continuing education as the preferred modalities for training. While barriers related to time, cost and travel are nearly the same as those identified in the needs assessment surveys of AMCHP, 1992 and Alexander et al. 2002, (6-7) there have been definite shifts in preferred training strategies on the part of the state Title V public health workforce. Interestingly, the preferred continuing education strategy of skills-building workshops at conferences speaks to the success of this approach. However, the apparent contradiction in the responses provided 
9-23-10

reflects that skills building workshops at national meetings are an excellent venue for capacity building for those who can attend (typically, directors or designated staff such as MCH epidemiologists), but not a meaningful approach to continuing education for the many staff members who are unable to travel to such meetings.

To address the ongoing as well as emerging training needs of the state Title $\mathrm{V}$ workforce as well as their preferences for the receipt of training, it is important to consider the infrastructure currently available to do so and to explore whether and the extent to which this infrastructure needs to be retooled, restructured, and/or enhanced. The entities specifically charged with training the $\mathrm{MCH}$ public health workforce are the 12 Schools of Public Health funded by the MCHB to offer both masters and doctorate level degree programs (Appendix A). Importantly, MCHB requires all SPH Training Programs to offer some form of continuing education as well as technical assistance. Continuing education activities are delivered through a variety of modalities including local and regional workshops, informational newsletters, web-based modules as well as local/regional/national conferences.

As noted above, MCHB also sponsors special grant programs designed to reach $\mathrm{MCH}$ practitioners. Currently, the Bureau funds two MCH certificate programs, six continuing education grants and seven distance learning grants that sponsor a mix of national/regional conferences, and webbased learning initiatives. About half of these trainings focus on broad leadership and or public health skills and knowledge base; the other efforts are topically focused (e.g., oral health, suicide prevention). One of the larger MCHB grants that aims to build the capacity of the MCH public health workforce is the MCH Leadership Institute, which imparts broad basic MCH knowledge and leadership skills through two intensive on site sessions and year long web-based skills development modules. 
MCHB also funds resource centers that offer a variety of training materials and opportunities for continuing education that can be used to enhance the knowledge base and professional development of Title V staff (Appendix B). Beyond those presented by MCHB-funded resource centers, MCHB also routinely sponsors “data speaks” and webcasts presented in collaboration with MCH partner organizations.

While there are a wide range of graduate education and continuing education efforts funded by MCHB designed to meet the current and emergent needs of the MCH public health workforce, given the changing needs expressed by state $\mathrm{MCH}$ leaders as well as their preferences for receiving additional training, it is important that current and future GE and CE approaches (both content and structure) be appropriately aligned to meet these needs. With respect to graduate education, although MCH programs in schools of public health and related institutions (e.g., schools of social work) must continue to educate masters and doctoral level MCH students in both the fundamental MCH knowledge base as well as key skills, the data presented here suggest that graduate MCH education in public health needs to not only emphasize the generation of data (i.e., research and analysis skills) but also to place increased emphasis on the translation of data into evidence based public health practice. This type of effort may require enhanced interaction of students and faculty with MCH practice partners as translation requires the movement from data/research findings to implementation. The findings of this assessment also suggest an increased emphasis on critical thinking skills; while this is generally the hallmark of graduate education activities, it is possible that these efforts could be conducted in conjunction with practice partners (e.g., on-line journal clubs or learning collaborative of MCH academe and practice partners).

In response to the preference for blending learning in graduate education, there may likely be a need for MCH training programs in SPH to increase distance education efforts. Although distance education has been embraced by many schools of public health, the emphasis in graduate education has 
9-23-10

historically been on face to face in-person learning. As such, the modality embraced by SPHs for distance learning may be particularly important for both learner and teacher. The current literature (910) provides support for distance education approaches in which there is some verbal interaction with the instructor (e.g., web camera approaches). These interactive approaches are likely to be even more salient when there is increased emphasis on interpretation, translation, and use of evidence in practice.

With respect to continuing education, the findings of the survey data presented here suggest that a mix of educational modalities will be necessary to meet emerging needs. Over the last decade, skills building workshops have been institutionalized at the four main meetings for MCH public health practice (AMCHP, CityMatCH, Maternal, Infant and Child Health Epidemiology Program (MICHEP), and the MCH Leadership Retreat). Survey respondents gave their approval to this approach, with 63\% of respondents stating that this was their preferred method of continuing education. However, given the expressed need of survey respondents for translation type skills which range from understanding and interpreting the literature to scientific and policy writing, there may be a need for more ongoing instruction and support beyond what can be provided in time-limited skills building workshops.

As such, it may be both necessary and desirable to combine face to face skills building workshops at meetings with follow-up activities delivered via distance modalities, ranging from teleconferences to the use of second generation web technologies such as blogs or wikis and other social networking tools. While national meetings remain the most preferred strategy for training, regional meetings also were among the top three modalities preferred; convening staff for training at the regional level also may serve to address time and cost barriers noted in survey responses. In addition, for individuals not able to attend any in person meetings or workshops an array of interactive or self-paced learning opportunities need to be available through both web and teleconference approaches. One approach that is responsive to adult learning needs is Learning Collaboratives in 
9-23-10

which peers from various agencies (in this case across states) come together to tackle current work challenges, share experiences, discover new strategies to tackle a problem, and provide feedback and resources once new interventions or enhancements are implemented. These learning collaboratives, as suggested above, can engage both members of academe and practice and can support the concept of enhancing critical thinking skills among all members. Another example of a distance method that is responsive to adult learning needs is ‘just in time’ learning, where short online modules address common issues, and shorten the time between learning and application. Social networking tools also allow managers to engage with more diverse groups to address emerging issues and can provide a way to better connect older and younger generations in the workplace.

We believe that the changes in the training needs of the MCH public health workforce described here are due in part to the effectiveness of specific MCHB and CDC training initiatives carried out over the last twenty five years, particularly those focused on data and analytic capacitybuilding. One of the most pivotal state analytic capacity building initiatives, for example, was the creation of the CDC/HRSA MCH Epidemiology Program (MCHEP), which in 1986 began assigning MCH epidemiologists to serve as senior scientists to provide state MCH programs with the analytic leadership necessary to engage in data-based decision-making. (11-12) In concert with and parallel to this investment, other data capacity building initiatives sponsored by the federal government and their partners such as the Association of State and Territorial Health Officials (ASTHO) and the Council of State and Territorial Epidemiologists (CSTE) have included ongoing delivery of both face-to-face and distance-based trainings, and publication of analytic methods workbooks, briefs, tools and modules. $(13,14)$ Also prominent in these data efforts has been supplemental Title V funding to states through the State Systems Development Initiative (SSDI) to facilitate improvement in components of the data infrastructure. 
The MCH workforce assessment described here has a number of limitations. The survey asked about training needs of program staff as a group. As such, we are unable to report on specific staff characteristics and possible associations between needs cited and demographic characteristics, academic preparation or years of experience. In addition, Title V programs were asked to reflect on training needs primarily in relation to their mid- and senior-level staff and our findings do not necessarily reflect the preferences of more junior MCH professionals. Finally, our findings are limited to a very specific part of the maternal and child health workforce and do not account for local training needs and concerns in either the public or private sectors.

Given the multiple opportunities for both continuing and graduate education available to the MCH workforce, developing an approach that attempts to maximize resources by making them more readily available and accessible to all MCH professionals will be increasingly important. Two essential aspects of such an approach include: 1) the creation of a web-based MCH workforce training resource inventory to delineate all training options and to provide recommendations and criteria for each offering based on the content and skills included; and, 2) the development of personalized assessment tools that assist individuals in understanding their knowledge and skills deficiencies in relation to their current job responsibilities. With these two approaches in place, a broker, either an institution or a learning management system, can then assist $\mathrm{MCH}$ professionals in matching their learning needs with available offerings.

Also critical will be the commitment of State Title V Directors to workforce training efforts. Title V Directors need to set the tone within their State programs and model expected training behaviors. Individuals hired into state-level positions come with diverse educational and work experiences, few with formal MCH training. Multiple strategies are available to program leaders, including annual assessments of needed staff training and development of a State Title V Program plan 
9-23-10

in each state that ensures training is carried out to build MCH capacity; group trainings as part of routine staff meetings or retreats, which can enhance working relationships and build a team environment; and integrating MCH competencies into individual staff's daily work responsibilities and annual performance plans. Finally, it will be important to track progress in building MCH capacity through venues like annual program capacity assessments and documenting staff's training-related accomplishments in the context of individualized performance evaluations.

\section{CONCLUSIONS}

Reviewing the host of initiatives aimed at increasing MCH state data collection and analysis capacity is informative, particularly because although states now appear to have substantially increased data capacity attributable to this investment, they also appear to have insufficient resources and skills needed to utilize/translate these data for/into program and policy change and intervention. (15) Therefore, we believe that a similar targeted and long-term investment focused on data use, interpretation and translation is now necessary.

While the opportunity for change and improvement as the $\mathrm{MCH}$ field continues to grow and mature is enormous, it is also important that these changes rest on the solid fundamentals that are the backbone of $\mathrm{MCH}$ public health education. The challenge will be to incorporate new emphases and approaches while ensuring that all members of the $\mathrm{MCH}$ workforce are grounded in elemental $\mathrm{MCH}$ knowledge and planning cycle skills.

Faced with an aging workforce, severely restricted travel, a mind boggling array of new technological innovations, and evolving roles and demands in public health, MCH professionals need to use all creative means at their disposal to learn throughout their careers, and connect with peers and partners for creative problem solving. A variety of in-person and distance education methods can help 
9-23-10

achieve these goals. Much of the content expertise already exists, we now need to seize the opportunity to develop mechanisms to regularly assess workforce needs, utilize technology to develop innovative collaborative learning experiences that both improve skills and also foster a sense of MCH community, and evaluate our efforts. 
9-23-10

\section{ACKNOWLEDGMENTS}

The authors appreciate the efforts of the AMCHP Workforce Development Committee who assisted with the survey design and guided presentation of these results. We also are grateful to the state Title V programs for their participation in the survey. This project was partially supported through Maternal and Child Health Bureau, HRSA Grant U01MC00001 to the Association of Maternal and Child Health Programs (AMCHP) and HRSA, MCHB, funding to the Johns Hopkins Bloomberg School of Public Health for its MCH Leadership Training Program. Survey development and analysis were supported through AMCHP membership dues. 
9-23-10

\section{REFERENCES}

1. ASTHO. (2008). 2007 State Public Health Workforce Survey Results. Arlington, VA: Association of State and Territorial Health Officials.

2. HRSA. (2005). Public Health Workforce Study. Washington, DC: U.S. Department of Health and Human Services, Health Resources and Services Administration.

3. NACCHO. (2007. The Local Health Department Workforce: Findings from the 2005 National Profile of Local Health Departments Study. Washington, DC: National Association of County and City Health Officials.

4. NACCHO. (2009). NACCHO Survey of Local Health Departments' Budget Cuts and Workforce Reductions Washington, DC: National Association of County and City Health Officials.

5. Levi J., Kaiman S., Juliano C., and Segal L. (2008). Blueprint for a Healthier America: Modernizing the Federal Public Health System to Focus on Prevention and Preparedness. Washington, DC: Trust for America's Health. http://healthyamericans.org/assets/files/Blueprint.pdf.

6. AMCHP. (1992). Meeting Needs, Building Capacities: State Perspectives on Graduate Training and Continuing Education Needs of Title V Programs. Washington, DC: Association of Maternal and Child Health Programs.

7. Alexander G., Chadwick C., Slay M., Petersen D., and Pass A. (2002). Maternal and Child Health Graduate and Continuing Education Needs: A National Assessment. Maternal and Child Health Journal 6(3):141-149.

8. Grason H., Guyer B. (1995). Public MCH Program Functions Framework: Essential Public Health Services to Promote Maternal and Child Health in America. Baltimore, MD: Johns Hopkins Child and Adolescent Health Policy Center. 
9-23-10

9. Rovai, A.P. and Jordan H.M. (2004). Blended Learning and Sense of Community: A comparative analysis with traditional and fully online graduate courses. International Review of Research in Open and Distance Learning, 5(2).

10. Swan, K. (2002). Building Learning communities in Online Courses: the importance of interaction. Education, Communication \& Information, 2(1).

11. Rochat R., Atrash H., and Handler A. (1999). Observations from the CDC: developing maternal and child health epidemiology capacity in state and local health departments. $J$ Women's Health 8(9):1135-9.

12. Handler, A., Geller, S., and Kennelly, J. (1999). Effective MCH Epidemiology in State Health Agencies: Lessons from an Evaluation of the Maternal and Child Health Epidemiology Program (MCHEP). Maternal and Child Helath Journal 3(4):217-224.

13. Handler A., Kennelly J., and Rosenberg D. (2001). Building the Analytic Capacity of the State Maternal and Child Health Workforce-A History of the HRSA/MCHB Academic Partnership. Journal of Public Health Management Practice, 7(4):83-90

14. Ruderman M., Grason H., and Hess C. (2005). Five Years of Experience with Capacity Assessment for State Title V (CAST-5). Baltimore, MD: Women’s and Children’s Health Policy Center, Johns Hopkins Bloomberg School of Public Health.

15. Rosenberg D., Kennelly J., Herman-Roloff A., Rochat R., and Handler A. (2008). Maternal and Child Health Epidemiology in State Health Agencies: Guidelines for Enhanced Functioning. Chicago, IL: University of Illinois at Chicago School of Public Health. 